\title{
Micro-channels in the mastoid anatomy. Indications of a separate blood supply of the air cell system mucosa by micro-CT scanning
}

Olivier Cros, Magnus Borga, Elin Pauwels, Joris J. J. Dirckx and Michael Gaihede

\section{Linköping University Post Print}

\section{Tweet}

N.B.: When citing this work, cite the original article.

Original Publication:

Olivier Cros, Magnus Borga, Elin Pauwels, Joris J. J. Dirckx and Michael Gaihede, Microchannels in the mastoid anatomy. Indications of a separate blood supply of the air cell system mucosa by micro-CT scanning, 2013, Hearing Research, (301), 60-65.

http://dx.doi.org/10.1016/j.heares.2013.03.002

Copyright: Elsevier

http://www.elsevier.com/

Postprint available at: Linköping University Electronic Press

http://urn.kb.se/resolve?urn=urn:nbn:se:liu:diva-92813 


\section{Micro-channels in the mastoid anatomy.}

Indications of a separate blood supply of the air cell system mucosa by micro-CT scanning.

Olivier Cros ${ }^{1,2,3}$, Magnus Borga ${ }^{2,3}$, Elin Pauwels ${ }^{4}$, Joris JJ Dirckx ${ }^{5}$, and Michael Gaihede ${ }^{1}$.

${ }^{1}$ Department of Otolaryngology, Head and Neck Surgery, Aalborg University Hospital, Denmark,

${ }^{2}$ Department of Biomedical Engineering, Medical Informatics, Linköping University, Sweden,

${ }^{3}$ Center for Medical Image Science and Visualization, Linköping University, Sweden,

${ }^{4}$ Centre for X-ray Tomography, Department of Physics and Astronomy, University of Ghent, Belgium and,

${ }^{5}$ Laboratory for Biomedical Physics, University of Antwerp, Belgium

Corresponding author:

Name: Olivier Cros.

E-mail address olivier.cros@liu.se.

Address: Department of Biomedical Engineering, Medical Informatics, Linköping University, SE-581 85 Linköping, Sweden.

Tel: +46 (0)13286724.

Fax: +46 (0)13 101902 .

Abbreviations: $\mathrm{ME}=$ middle ear; $\mathrm{TM}$ = tympanic membrane; 


\begin{abstract}
The mastoid air cell system has traditionally been considered a passive role in gas exchange and pressure regulation of the middle ear possibly with some acoustic function. However, more evidence has focused on the mucosa of the mastoid, which may play a more active role in regulation of middle ear pressure.
\end{abstract}

In this study we have applied micro-CT scanning on a series of three human temporal bones. This approach greatly enhances the resolution (40 to $60 \mu \mathrm{m}$ ), so that we have discovered anatomical details, which has not been reported earlier. Thus, qualitative analysis using volume rendering has demonstrated notable micro-channels connecting the surface of the compact bone directly to the mastoid air cells as well as forming a network of connections between the air cells. Quantitative analysis on 2D slices was employed to determine the average diameter of these micro-channels $(158 \mu \mathrm{m}$; range $=40$ to $440 \mu \mathrm{m})$ as well as their density at a localized area (average $=75 \mathrm{~cm}^{-2}$; range $=64$ to $97 \mathrm{~cm}^{-2}$ ).

These channels are hypothesized to contain a separate vascular supply for the mastoid mucosa. However, future studies of the histological structure of the micro-channels are warranted to confirm the hypothesis. Studies on the mastoid mucosa and its blood supply may improve our knowledge of its physiological properties, which may have important implications for our understanding of the pressure regulation of the middle ear.

Keywords: human temporal bone, micro-tomography, volume rendering, micro-channels, vascularization, mastoid, mucosa. 


\section{Introduction}

The middle ear (ME) of the human temporal bone contains both the mastoid and the tympanum. Whereas the mastoid consists of a larger series of connected air filled cells divided by bony septae, the tympanum can be considered a single air cell which contains the ossicles. These structures are important for the transfer of sound pressures from the ear canal and tympanic membrane (TM) to the inner ear; thus, the function of the tympanum mainly relates to sound conduction. However, the physiology of the mastoid is not entirely understood although its structural properties may indicate more functions. The numerous bony septae separating the mastoid into smaller air cells may imply an acoustic function by reducing resonances and increasing hearing sensitivity for low frequencies (Fleischer, 2010). More well known aspects of the mastoid function relate to ME pressure, gas exchange, and otitis media (Sadé \& Ar, 1997; Doyle, 2000).

The mastoid cellular structure greatly enhances its surface area-to-volume ratio (AV-ratio); based on the analysis of clinical CT scannings the AV-ratio is on average $16 \mathrm{~cm}^{-1}$ (Park et al., 2000). Moreover, the mastoid mucosa is relatively thin with a flat cuboidal surface epithelium and a rich superficial vascular supply; the distance from its surface to the underlying vascular structures is smaller than found for the mucosa of the tympanum (Ars et al., 1997). These structural findings suggest that mastoid gas exchange is favored both by a high AV-ratio as well as a short mucosal diffusion distance and high perfusion (Ars et al., 1997; Park et al., 2000).

We have previously investigated methods of image analysis on clinical CT scannings of the mastoid in order to describe methods for semi-automatic determination of its surface area and volume; however, we found that their resolutions may be too low for detection of the smallest 
air cells. Consequently, we supplemented these investigations by applying micro-CT scanning on a smaller series of temporal bones. By first visual inspection of these data we found numerous micro-channels which have not been described earlier in the literature. The current study reports on the structure of these channels, because they are likely to represent a separate blood supply for the mastoid mucosa. This may have important implications for the understanding of the ME physiology including its pressure regulation and gas exchange.

\section{Material and methods}

\subsection{Data preparation}

Three human temporal bones were used in this study; they were preserved in alcohol after removal of their surface soft tissues and kept in our laboratory for otosurgical training purposes. Scanning of the bone specimens was performed using a custom-built micro-CT scanner available at the Department of Physics and Astronomy, Ghent, Belgium (Masschaele et al., 2007).

Before the scanning process, each bone specimen was carefully placed in a plastic cup on a computer-controlled rotation table in order to avoid displacements during rotation. Based on the sample size and composition the optimal acceleration voltage of the X-ray source was set to $120 \mathrm{KeV}$ with $3 \mathrm{~mm}$ of aluminum of beam filtration. A full cone beam scan (ranging from $0^{\circ}$ to $360^{\circ}$ ) was recorded with 2 seconds of exposure per projection to ensure a high signal to noise ratio. A stack of cross-sections (2D slices in TIFF format) was then reconstructed using the in house developed Octopus package (Vlassenbroeck et al., 2007); additional aspects of application have been described by Buytaert et al. (2011). Because the TIFF format does not contain information about slice thickness and physical location of the slice in relation to the full 3D volume, the 2D slices were subsequently converted to DICOM format and updated with this information. Thus, the cross-sections were then assembled as a stack of slices along 
the Z-dimension to form a 3D volume; this conversion did not alter the data otherwise.

Basic information of the generated scans for the three bone specimens are presented in Table 1. The data size of these scans varied between 3.6 and 6.5 GB. In order to reduce the subsequent processing time these original data were cropped and only the relevant parts of the scans were kept (Table 2); this procedure resulted in data size ranging from 1.3 to 3.5 GB. Finally, due to technical reasons, scannings of the specimens were not performed in the coordinate system which is used in conventional clinical scannings (axial, coronal, and sagital). Therefore, a 3D affine transformation was applied for each specimen volume to ensure realignment of the data into this conventional system.

Table 1. Original dimension of each bone dataset.

\begin{tabular}{ccccc}
\hline Bone & $\begin{array}{r}\text { In-plane resolution } \\
\text { (pixels) }\end{array}$ & Number of slices & Isotropic voxel size $(\mu \mathrm{m})$ & Scan volume \\
& $2008 \times 2008$ & 1737 & & $(\mathrm{~mm})$ \\
\hline 1 & $1708 \times 1708$ & 1774 & $\sim 40$ & $79.3 \times 79.3 \times 68.6$ \\
2 & $1360 \times 1658$ & 1737 & $\sim 60$ & $94.4 \times 94.4 \times 98.1$ \\
3 & & & & $80.8 \times 80.8 \times 98.5$
\end{tabular}

Table 2. Cropping of the datasets.

\begin{tabular}{lccc}
\hline Bone & $\begin{array}{c}\text { In-plane resolution } \\
\text { (pixels) }\end{array}$ & Number of slices & Scan volume (mm) \\
\hline 1 & $1560 \times 1400$ & 1737 & $61.6 \times 55.3 \times 68.6$ \\
2 & $1346 \times 1200$ & 1300 & $74.4 \times 66.3 \times 71.8$ \\
3 & $1000 \times 1548$ & 900 & $59.4 \times 92 \times 53.5$ \\
\hline
\end{tabular}




\subsection{Quantitative analysis based on 2D images}

In order to provide a quantitative description of the micro-channels within the mastoid air cells, a region of interest (ROI) was defined posteriorly to the ear canal on a 2D axial slice in each bone specimen. This part was in the middle of the mastoid and decided to be a representative area; the size of the ROI was $6 \times 6 \mathrm{~mm}$ (Fig. 1). The analysis was performed using Matlab (MathWorks, Natick, MA), where the content of the respective ROI's was segmented into a binary representation with pixel data representing bone in a first class and all remaining pixel data in a second class. All unwanted structures such as larger air cell cavities or structures emanating from noise, air cavities hampered by partial volume effects, or irregularities at the border of the bone were excluded from the analysis. The remaining air cavities were defined as micro-channels. The number of micro-channels per ROI was then determined as well as their diameter through an eigenanalysis by computing the minor axis of their corresponding ellipsoid.

\section{Figure 1}

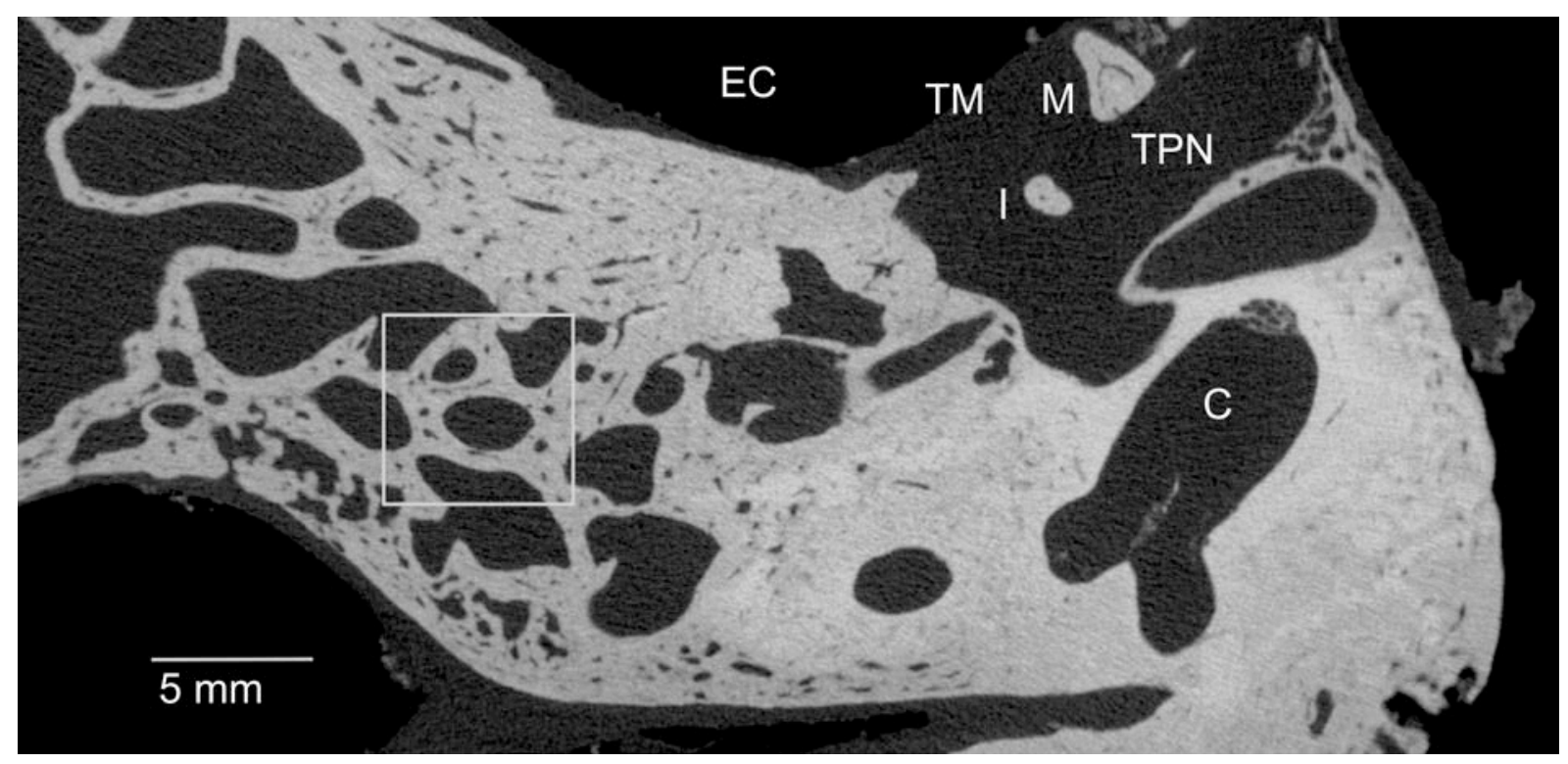




\subsection{Qualitative analysis based on volume rendering}

Visualization of the 3D structure of the micro-channels was performed by direct volume rendering using MevisLab, which is a free scientific graphical software. This software provided a transfer function which could be used to visualize the channels. A transfer function maps the scalar data from the original data into a color and opacity (Drebin et al., 1988). Figure 2 shows a transfer function (black piecewise linear function in front of the dark gray area). The dark gray area, known as a histogram, represents the occurrence of each gray value present in the data. This curve has three overlapping bell shapes corresponding to air, soft tissue, and bone (Drebin et al., 1988). The transfer function was used to adjust which part of the histogram needed to be displayed; thus, setting of the transfer function allowed the visualization of a limited part of the data reflecting a limited part of the tissues.

\section{Figure 2}

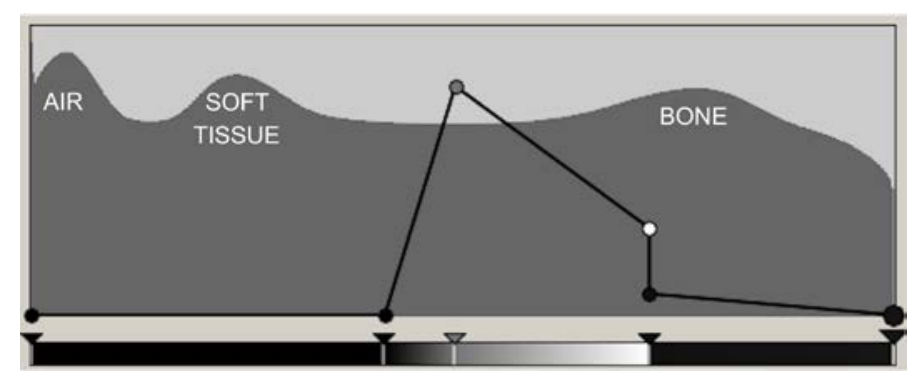

Because we were interested in the bone structures of the images, the transfer function was set, so that the air and the mucosa were compiled into one category, while the bone remained a second category. Thus, by enhancing the transfer function at the area between bone and the rest (air and soft tissues), the transitional voxels could be displayed; this is illustrated in Figure 2 by the peak of the transfer function (dark line). Transitional voxels illustrated the voxels found at both the transition from air to bone and bone to air, and hence, by this approach all bony surfaces could be displayed. Further, this process also resulted in hiding of all the soft 
tissues from the bone specimen.

Finally, the 3D and depth perception of the images were enhanced by truncating and directly assigning a slow-varying down-slope to the transfer function, as well as control points of the transfer function were assigned a grey level. Altogether, the steps of the transfer function were empirically adjusted and optimized for each individual bone (Fig. 2). These steps only changed the way the data were visualized, and hence, they did not alter the content of the image data.

\section{Results}

\subsection{Quantitative analysis based on 2D images}

The three mastoid bones appeared with normal pneumatization of the air cell system without sclerotic changes seen in diseased mastoids (Fig. 1). Thus, the three bones were considered to represent normal ME's. Table 3 depicts the diameter and the density of the channels; their mean diameter was $158 \mu \mathrm{m}(\mathrm{SD}=74 \mu \mathrm{m})$ with a range between 40 and $440 \mu \mathrm{m}$. The number of channels within the ROI's of the three specimens varied from 23 to 35 (mean $=27$ ), while their density varied between 64 and $97 \mathrm{~cm}^{-2}$ (mean $=75 \mathrm{~cm}^{-2}$ ).

Table 3. Distributions of the dimensions of the micro-channels from the ROI of each bone and their densities from the sampled areas $(6 \times 6 \mathrm{~mm})$.

\begin{tabular}{ccccc}
\hline Bone & Mean diameter $(\mathrm{SD})(\mu \mathrm{m})$ & Range $(\mu \mathrm{m})$ & No. of channels & Density $\left(\mathrm{cm}^{-2}\right)$ \\
\hline 1 & $122(73)$ & 40 to 320 & 35 & 97 \\
2 & $189(78)$ & 110 to 440 & 23 & 64 \\
3 & $162(70)$ & 60 to 240 & 23 & 64 \\
Overall & $158(74)$ & 40 to 440 & 27 & 75 \\
\hline
\end{tabular}




\subsection{Qualitative analysis based on volume rendering}

Figure 3 gives an overview of a temporal bone. The image is shown with an oblique lateral view in order to enhance the $3 \mathrm{D}$ perception of the structures. More straight parallel microchannels are seen traversing the compact bone; they appear on the surface of the bone as small indentations and more of these indentations are seen on the surface in the lower part of the inserted oval; in addition, more channels can be seen connecting to airs cells at the upper part of the inserted oval. The indentations and the channels appear mostly in the vicinity of the posterior part of the ear canal, and they become less frequent or absent at the posterior part of the bone, where also no air cells are found below the surface (inserted square). Moreover, micro-channels can be seen between the air cells; these channels have more irregular structure following the shape of the air cells. These properties could be confirmed by interactive examination of the full volumes as well as further details could be revealed by increasing the magnification; selected examples have been presented in Figures 4-6. 


\section{Figure 3}

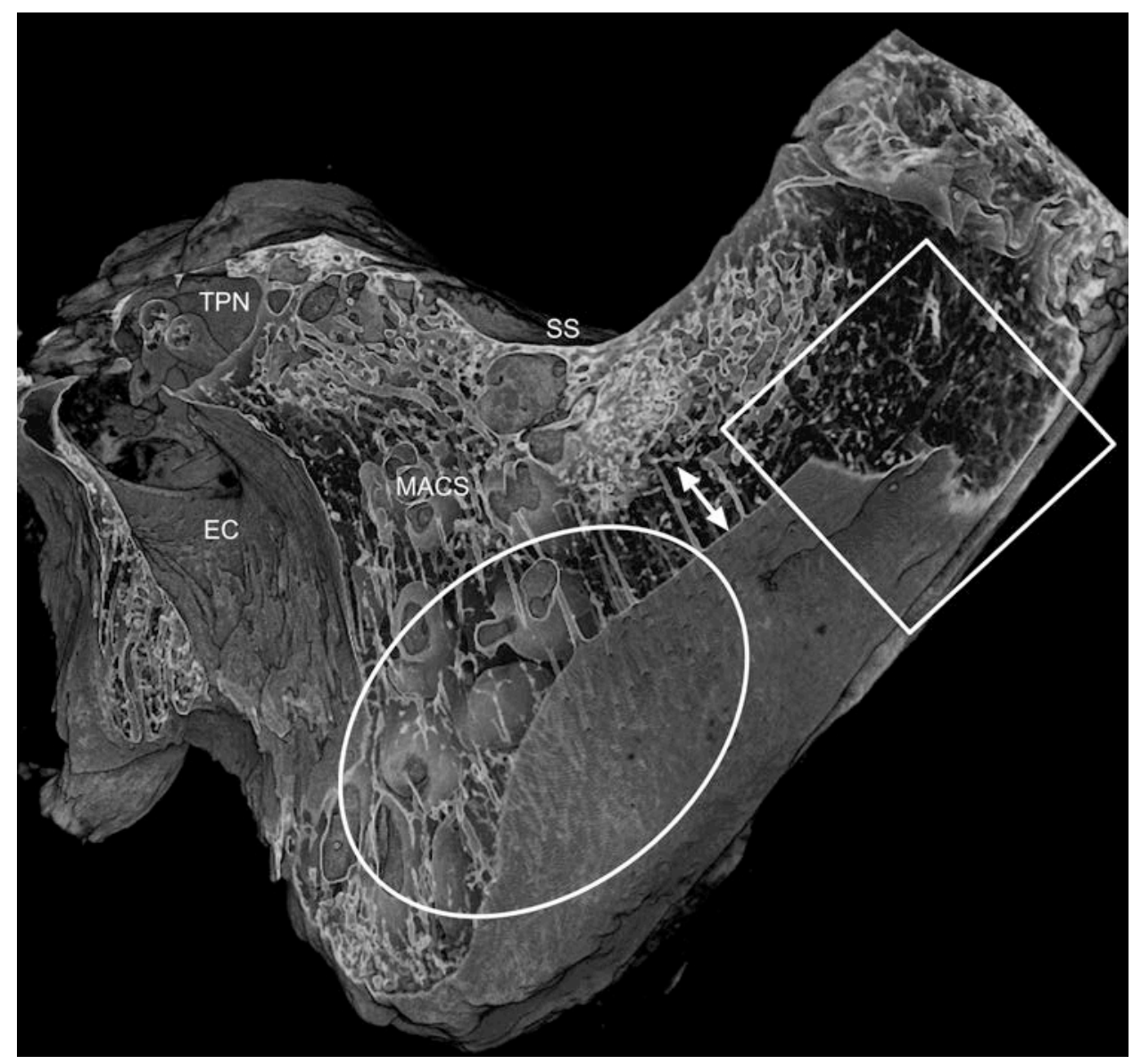

In Figure 4, multiple tiny holes or indentations in the surface of the compact bone can be seen at the posterior ear canal wall extending all the way to the level of the tympanum; in some cases it can be seen that the indentations are connecting directly with the underlying channels. In Figure 5 similar connections between indentations and channels can be seen, but in addition two of these channels are connected directly to an air cell, whereas in total at least four channels are connected to this air; this image is from the supero-posterior wall of the ear canal. Figure 6 displays a direct connection between the outer surface of the bone at the tip of the mastoid and a lower air cell by a particularly long channel; below this air cell the bone structure is found trabecular. The longer channel can be seen to branch into more channels close to the air cell, but also along its course through the trabecular area. 
Further interactive examinations of the adjacent images confirmed these properties for all three bones: 1) all surface indentations were connected to underlying channels running in parallel through the compact bone, 2) air cells were in all cases connected to more channels, where some connected directly to the surface indentations, 3) irregular channels were found between the air cells, where channel connections between cells were found, and 4) the channels occasionally branched in the area between the air cells as well as in trabecular and compact bone.

\section{Figure 4}

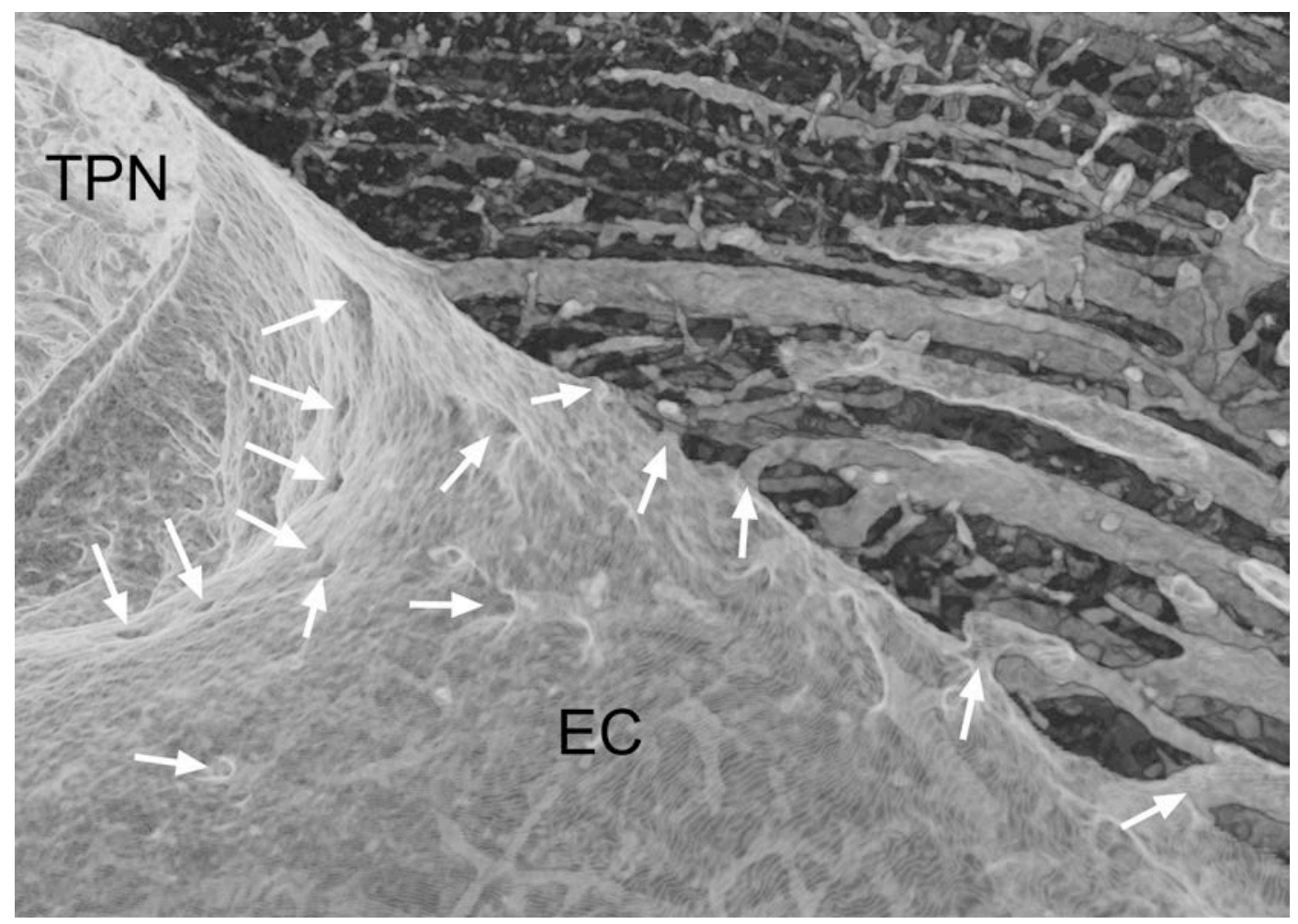




\section{Figure 5}

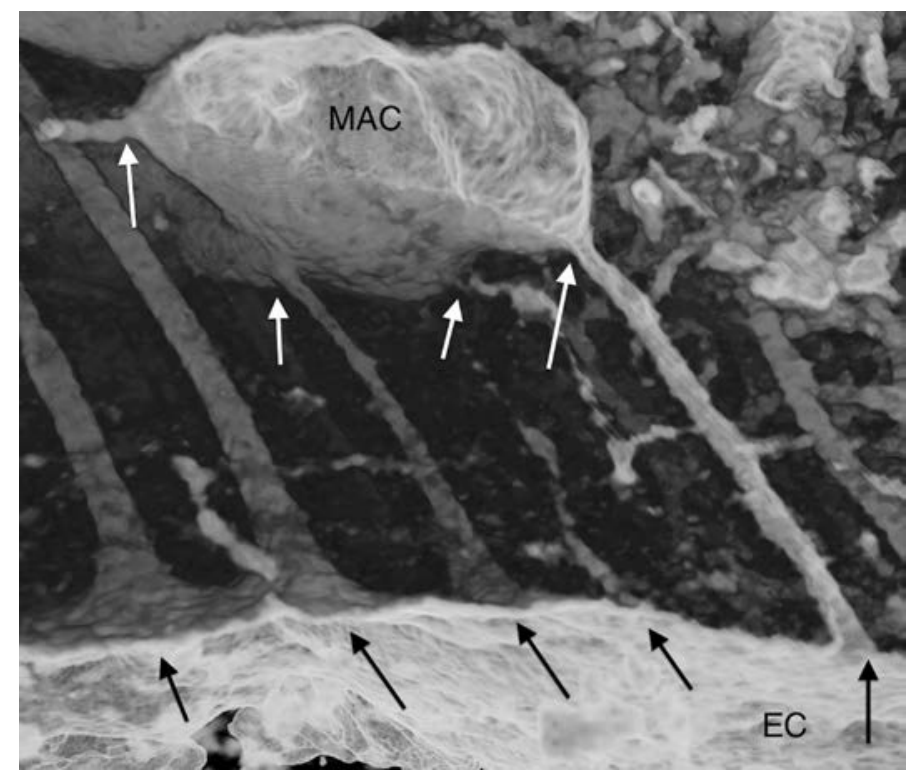

Figure 6

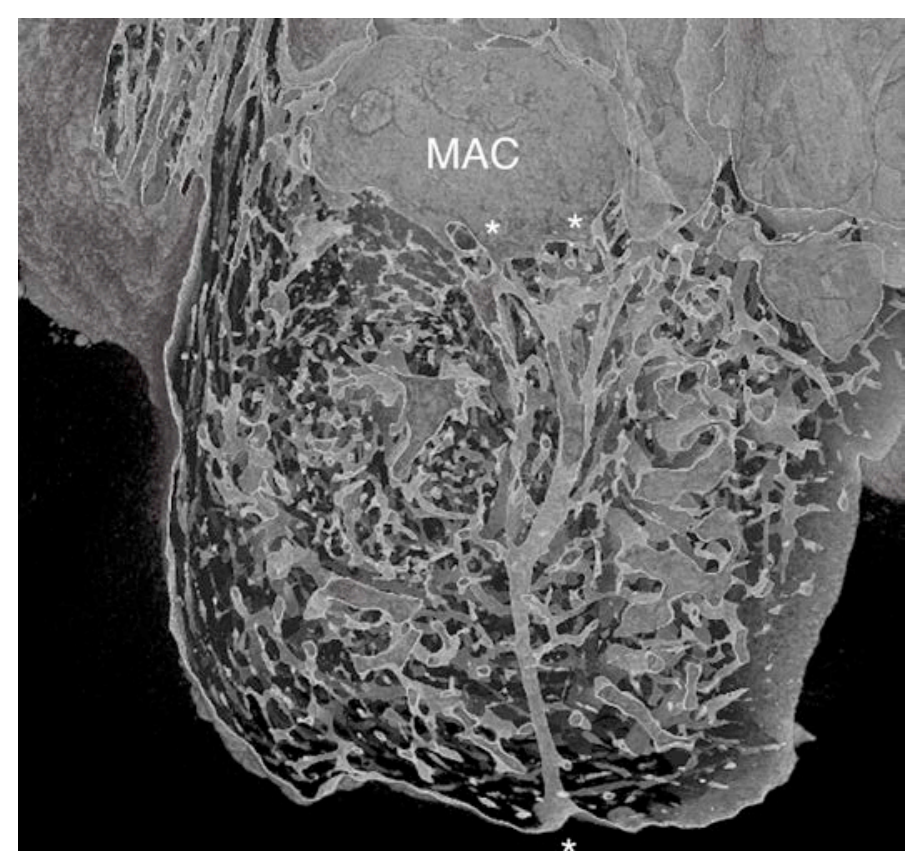

\section{Discussion}

The image analysis of the mastoid carried out in this study was based on micro-CT-scanning.

This modality greatly improves the resolution of images, and it has already been applied in

ME research modeling the anatomy of ME itself as well as the ossicular chain (Lee et al., 
2010; Salih et al., 2012); however, micro-CT scannings applied in research of the mastoid has not been reported earlier.

Similar to high-resolution clinical scannings of the temporal bone, the present micro-CT scanning images also clearly demonstrated the air filled cellular structure of the mastoid, but in addition we have consistently discovered notable micro-channels. The dimension of the micro-channels was determined by quantitative analysis of the 2D images showing a mean diameter of $158 \mu \mathrm{m}$ with a range between 40 and $440 \mu \mathrm{m}$ (Table 3). The resolution of our micro-CT scannings varied between 40 to $60 \mu \mathrm{m}$ (Table 1); this corresponded to the resolution reported for the human ossicles of $34 \mu \mathrm{m}$ (Salih et al., 2012). In practice, the resolution depended on the size of the specimen, so that smaller specimens resulted in larger resolution, and vice versa. Altogether this means that the smallest channels were at the border of the resolution of the micro-CT scannings, whereas the larger channels were well below the resolution of clinical CT scannings $(\approx 600 \mu \mathrm{m})$. Thus, these channels could not be detected by conventional clinical scannings, and in accordance, we have not found any descriptions of them in the contemporary literature on temporal bone anatomy (Gulya, 2007; Lane \& Witte, 2009). Moreover, similar occurrence of micro-channels has not been found in other bone tissues at our laboratory in Ghent (unpublished observation). Therefore, we consider the microchannels a new anatomical discovery.

The superficial indentations appeared quite dense which is most clearly illustrated in Figure 4. Since they were all connected to underlying micro-channels, these channels similarly appeared dense for a visual inspection; this was also confirmed by interactive examination of adjacent images. However, a quantitative measure of their density could only be obtained from the 2D images, where the mean density was $75 \mathrm{~cm}^{-2}$ (Table 3). This represented a lim- 
ited local estimate which may vary in other parts of the mastoid as well as it may be different in diseased mastoids. The detection of the channels could be influenced by their direction and shape, so that false positive channels may be detected. Similarly, partial volume effects and noise of the image could also result in false positive results. Finally, the detection of smaller openings, which may be related to the periphery of an air cell, could be avoided by examining the adjacent slices before counting the channels. Altogether, the current results formed a basis for future research with more complete analysis of their density in various regions in normal and diseased mastoids.

Volume rendering is a well established method in image analysis; this method does not alter the data, but enhances selected properties in order to illustrate the deeper 3D structure of image volumes (Drebin et al., 1988). Thus, whereas the method did not allow for quantitative analysis of the micro-channels, it demonstrated their 3D structure. The micro-channels showed two types: 1) parallel channels which emerged from indentations on the lateral surface of the temporal bone and inside the ear canal, and which traversed the compact bone; many connected directly to the air cells (Figs. 3, 4, 5); and 2) irregular shaped channels that seemed to follow the shapes of the bony septae between the air cells inside the mastoid, as well as they formed network by branching and connections between adjacent air cells (Figs. 3, 6). In addition, more channels were usually found connected to the individual air cells (Figs. $5,6)$. It should be noted that the transfer function enhanced transitional voxels found between one category (soft tissue and air) and in another (bone); thus, the bony surfaces of the structures were visualized. Whereas the preservation of the bones in alcohol may have altered the soft tissue structure, the bone structure was unlikely to be influenced by for instance shrinkage. This means our analysis focusing on the bony surfaces could be justified. 
The structural properties of the micro-channels resembled a vascular network. Considering their mean diameter of $158 \mu \mathrm{m}$, the micro-channels could contain both small arterioles and venules, which have diameters around $30 \mu \mathrm{m}$ and 10 to $60 \mu \mathrm{m}$, respectively (Boulpaep, 2009). Haversian canals are well known in bone providing a vascular supply for the tissue, but these canals are smaller up to $45 \mu \mathrm{m}$ in diameter and usually only containing capillaries (Cooper et al., 2003); thus, such canals were below the resolution of our method (40-60 $\mu \mathrm{m})$. However, the branches formed by micro-channels within the compact and the trabecular bone (Fig. 6) as well as in the septae between the air cells may be connected to smaller Haversian canals below our resolution; this would provide a vascular supply for the bone matrix itself. If the micro-channels contained a vascular network, it seemed to originate from the surface periosteum. Moreover, since all air cells were connected to more channels, it seemed reasonable to suggest that such a blood supply would be aimed at the mastoid mucosa.

In order to investigate the exact content of the micro-channels further histological studies are needed. Figure 8 shows a preliminary histological section from the surface of the mastoid at the posterior part of the ear canal similar to the image in Figure 4. This section clearly shows at the center a channel with a diameter of $133 \mu \mathrm{m}$ containing a venule and arteriole, respectively; in addition some connective tissue is found and the channel is surrounded by typical bone tissue. Thus, this preliminary investigation supported the idea that the micro-channels may contain vascular elements. Further investigations are obviously needed with for a detailed and systematic description of the mastoid structure in order to identify the true content of its micro-channels. Such investigations include histological examination of the mastoid with immuno-staining which can identify any vascular structures with high accuracy. Whereas volume rendering is unable to identify various types of tissue, this methodological still had the advantage that it demonstrated clearly the overall 3D structures; thus, it may also be a 
valuable approach for future research.

\section{Figure 7}

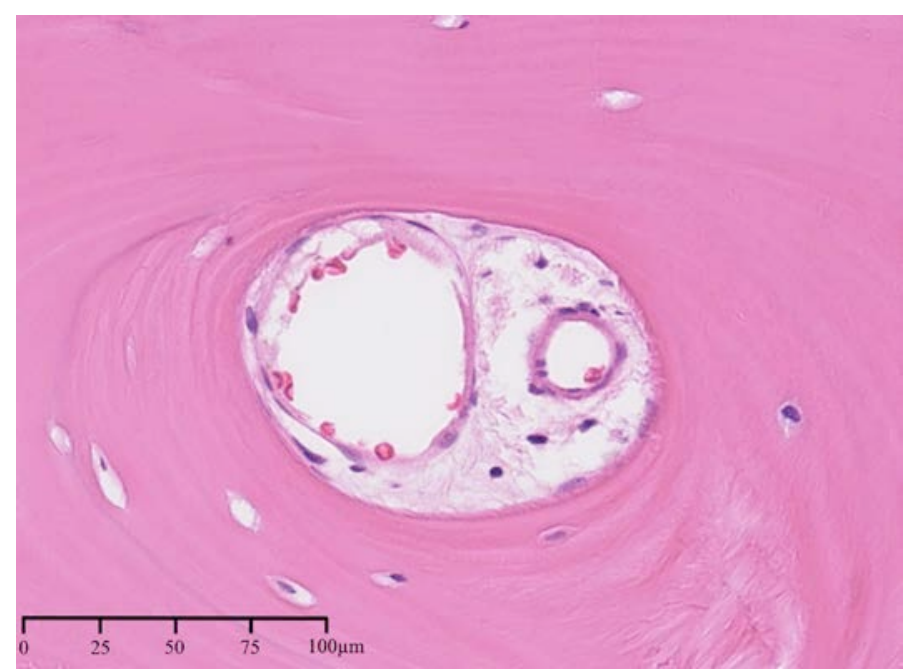

The normal adult mastoid develops after birth by expansion of air cells from the antrum; these air cells invade the mesenchymal tissue of the bone, and the mature fully aerated mastoid is normally reached between 14-16 years of age (Cinamon, 2009). It follows that the mucosa and its vascular supply would expand similarly from the antrum into the mastoid along with the formation of the air cells. Thus, if the micro-channels represented a vascular supply to the mastoid mucosa which originated from the surface periosteum, it seemed to constitute a separate or additional blood supply. Previously, rich vascular structures have been described in the mastoid mucosa, but the density of the vessels have not been reported by quantitative data (Ars et al., 1997). From a clinical experience, resection of the normal mastoid by drilling the air cells (mastoidectomy) does not give the impression of a rich blood supply, but this observation may be influenced by the fact that the mucosa is quite thin around $100 \mu \mathrm{m}$ (Ars et al., 1997). Moreover, despite cooling the surgical field with water, the drilling may still produce enough heat to cause a coagulation of the small vessels, so that no significant bleeding is encountered. 
From a basic point of view the current findings may be important, because a high vascularization enables both a high perfusion and effective changes in the vascular congestion. In terms of gas exchange and pressure regulation the role of the mucosal perfusion is still under debate (Marcusohn et al., 2010). Moreover, recent clinical investigations have shown systematic bidirectional short term counter-regulation of experimental changes in ME pressure which indicates that the mastoid may play an active role in pressure regulation (Gaihede et al., 2010). This may be accomplished by volumetric changes of the mucosa resulting from changes in its vascular congestion (Magnuson, 2003).

\section{Conclusions}

The current study demonstrated notable micro-channels in the temporal bone which connected the outer surface directly to the mastoid air cell system, but they also formed a network between the air cells; further, more channels were connected to the individual air cells. According to their dimensions and structure, these channels were hypothesized to represent a separate vascular supply for the mastoid mucosa. This may altogether indicate that the mastoid mucosa is well vascularized which may have functional implication related to regulation of ME pressure and gas exchange. The hypothesis needs to be investigated by future systematic and detailed histological studies of the mastoid.

\section{Acknowledgments}

The Spar Nord Foundation, The Obel Family Foundation, and The Research Administration at Aalborg Hospital, Aarhus University Hospital, generously supported this study. Giedrius Lelkaitis, M.D., Department of Pathology, and Simona Padurariu, M.Sc.D., Department of Otolaryngology, Head and Neck Surgery, Aalborg Hospital, are acknowledged for providing the histological specimen in Fig. 8 and revising the manuscript. 


\section{References}

Ars, B., Wuyts, F., Van de Heyning, P., Miled, I., Bogers, J., Van Marck, E., 1997. Histomorphometric study of the normal middle ear mucosa. Preliminary results supporting the gasexchange function in the postero-superior part of the middle ear cleft. Acta Otolaryngol. (Stockh), 117, 704-707.

Boulpaep, E., L., 2009. Arteries and veins. In Boron, W. F., Boulpaep, E. L. eds. Medical Physiology: a cellular and molecular approach. Saunders Elsevier, Philadelphia, PA, p 467469.

Buytaert, J. A. N., Descamps, E., Adriaens, D., Joris, J. J., 2011. The OPFOS microscopy family: high-resolution optical sectioning of biomedical specimens. Anat. Res. Int., 2012, 1-9. Cinamon, U., 2009. The growth rate and size of the mastoid air cell system and mastoid bone: a review and reference. European Archives of Oto-Rhino-Laryngology, 266, 781-786.

Cooper, D. M. L., Turinsky, A. L., Sensen, C. W., Hallgrimsson, B., 2003. Quantitative 3D analysis of the canal network in cortical bone by micro-computer-tomography. The anatomical record (part B: New Anat.), 247B, 169-179.

Doyle, W. J., 2000. Middle ear pressure regulation. In Rosowski JJ, Merchant SN eds. The function and mechanics of normal, diseased and reconstructed middle ears. The Hague, the Netherlands: Kugler, p3-21.

Drebin, R. A., Carpenter, L., Hanrahan, P., 1988. Volume rendering. Computer Graphics, 22, $4,65-74$.

Fleischer, G., 2010. Components for protecting and optimizing good hearing. In Theile, G., (Ed.). A contribution to the international convention of sound designers - Proceedings of the VDT 26th International Convention. 250-265. Bergisch-Gladbach, Germany, ISBN 978-39812830-1-3. 
Gaihede, M., Dirckx, J. J. J., Jacobsen, H., Aernouts, J., E., F., Søvsø, M., Tveterås., K., 2010. Middle ear pressure regulation - Complementary active action of the mastoid and the Eustacian tube. Otol. Neurotol., 31:603-11.

Gulya, A. J., 2007. Gulya and Schuknecht's anatomy of the temporal bone with surgical implications. New York: INFORMA Healthcare, 3rd Edition, p1- 356. ISBN: 0849375972 Lane, J. I., Witte, R. J., 2009. The temporal bone. An imaging atlas. Heidelberg: Springer, p1-109. ISBN: 3642022098.

Lee, D. H., Chan, S., Salisbury, C., Kim, N., Salisbury, K., Puria, S., Blevins, N. H., 2010. Reconstruction and exploration of virtual middle-ear models derived from micro-CT datasets. Hear. Res., 263, 198-203.

Magnuson, B., 2003. Functions of the mastoid cell system: auto-regulation of temperature and gas pressure. J. Laryngol. Otol., 117, 99-103.

Marcusohn, Y., Ar, A., Dirckx, J. J. J., 2010. Perfusion and diffusion limitations in middle ear gas exchange: The exchange of $\mathrm{CO}_{2}$ as a test case. Hear. Res., 265, 11-14.

Masschaele, B. C., Cnudde, V., Dierick, M., Jacobs P., Van Hoorebeke, L., Vlassenbroeck, J., 2007. UGCT: New x-ray radiography and tomography facility. Nucl. Instrum. Meth., A, 580, 266-269.

Park, M. S., Yoo, S. H., Hoon, D. H., 2000. Measurement of surface area in the human mastoid air cell system. J. Laryngol. Otol., 114, 93-96.

Sadé, J., Ar, A., 1997. Middle ear and auditory tube: Middle ear clearance, gas exchange, and pressure regulation. Otolaryngol. Head Neck Surg., 116, 499-524.

Salih W. H. M., Buytaert J. A. N., Aerts, J. R. M., Vanderniepen, P., Dierick, M., Dirckx, J. J. J., 2012. Open access high-resolution 3D morphology models of cat, gerbil, rabbit, rat, and human ossicular chains. Hear. Res., 284, 1-5. 
Vlassenbroeck, J. , Dierick, M., Masschaele, B., Cnudde, V., Van Hoorebeke, L., Jacobs, P., 2007. Software tools for quantification of X-ray microtomography at the UGCT. Nuclear Instruments and Methods in Physics Research, A, 580, 442-445. 


\section{Legends to Figures}

Fig. 1. Axial 2D section of a left temporal bone (bone 2) illustrating the mastoid posterior to the ear canal (EC). The inserted square defines the ROI of the mastoid air cells of $6 \times 6 \mathrm{~mm}$. TPN = tympanum; $\mathrm{TM}$ = tympanic membrane; $\mathrm{M}$ = malleus; $\mathrm{I}=$ incus; $\mathrm{C}$ = cochlea.

Fig. 2. Transfer function used in MevisLab. The transfer function is broken down into a piecewise linear function where control points can be interactively positioned (round dots on the black curve). The horizontal axis represents the grey levels from the data, while the vertical axis defines the opacity. The filled curve in the background (dark grey) represents the histogram of the data where three overlapping bell-shaped curves correspond to: air, soft tissue, and bone. The control points are placed in an empirical and optimal manner rendering only the transition voxels between air and bone; grey levels are assigned to each of these control points.

Fig. 3. Volume rendering image displaying the interface between the compact bone and mastoid (bone 2). Micro-channels are seen running in parallel through the compact bone which appears black; its thickness has been illustrated by the double-headed arrow. At the surface the channels appear as small indentations in the bone, and in the depth they run towards the air cells. These channels are primarily seen in the area around the posterior ear canal (oval marking), whereas they become less prominent or absent further posterior, where no air cells are found below the compact bone (square marking). TPN = tympanum; SS = sigmoid sinus; MACS = mastoid air cell system; EC = ear canal.

Fig. 4. Detailed volume rendering at the surface of the compact bone in the posterior wall of the ear canal (EC) (bone 1). Multiple indentations appear at the surface connecting to underly- 
ing micro-channels (white arrows); these channels are running in parallel through the compact bone. TPN = tympanum.

Fig. 5. Detailed volume rendering displaying the supero-posterior wall of the ear canal (EC) (bone 1). Micro-channels are directed in parallel (black arrows) towards the mastoid air cell (MAC). At least four channels are seen connected to the air cell; two channels can be seen connecting from the surface directly to the air cell.

Fig. 6. Coronal section at the tip of the mastoid (bone 1). One longer micro-channel is connecting the inferior surface of the bone to a lower mastoid air cell (MAC). Close to the air cell more branches are found entering the cell (white asterisks), as well as the channel gives more branches to the trabecular bone during its course to the air cell.

Fig. 7. Histological section of surface bone from the region behind the posterior ear canal (similar to Fig. 3; oval marking). The center contains a larger channel with a diameter of 133 $\mu$ m surrounded by bone tissue. Inside this channel two vessels are found: one larger vessel with thin walls (diameter $=65 \mu \mathrm{m}$ ), and one smaller vessel with thicker walls (diameter $=25$ $\mu \mathrm{m})$ corresponding to a venole and an arteriole, respectively. In addition some connective tissue surrounds the vessels (HE staining). 